\title{
Asymptomatic Submicroscopic Plasmodium Infection Is Highly Prevalent and Is Associated with Anemia in Children Younger than 5 Years in South Kivu/Democratic Republic of Congo
}

\author{
Yvette Lufungulo Bahati, ${ }^{1,2}$ Joris Delanghe, ${ }^{2}$ Ghislain Bisimwa Balaluka, ${ }^{3}$ Antoine Sadiki Kishabongo, ${ }^{4}$ and Jan Philippé ${ }^{2 \star}$ \\ ${ }^{1}$ Department of Pediatrics, Catholic University of Bukavu, Bukavu, Democratic Republic of Congo; ${ }^{2}$ Department of Diagnostic Sciences, Ghent \\ University, Ghent, Belgium; ${ }^{3}$ Department of Public Health, Catholic University of Bukavu, Bukavu, Democratic Republic of Congo; ${ }^{4}$ Department of \\ Clinical Biology, Catholic University of Bukavu, Bukavu, Democratic Republic of Congo
}

\begin{abstract}
One of the most important problems in controlling malaria is the limited access to effective and accurate diagnosis of malaria parasitemia. In the Democratic Republic of Congo (DRC), malaria is one of the leading causes of morbidity and mortality. The purpose of this study was to assess the prevalence of anemia and the relationship with asymptomatic submicroscopic Plasmodium infection. A cross-sectional study was carried out among 1,088 apparently healthy children aged between 6 and 59 months selected at random in the health zone of Miti Murhesa in South Kivu/DRC. Capillary blood was obtained for hemoglobin $(\mathrm{Hb})$ concentration measurement by Hemocue ${ }^{\circledR} \mathrm{Hb} 301$. Malaria detection was performed by microscopy and the loop-mediated isothermal amplification (LAMP) assay. Anemia was defined as $\mathrm{Hb}<11 \mathrm{~g} / \mathrm{dL}$. We applied the chi-square test for comparisons, and multiple logistic regression was used to identify the risk factors for anemia and submicroscopic Plasmodium infection. The prevalence of anemia was 39.6\%, and the prevalence of parasitemia was $15.9 \%$ and $34.0 \%$ using microscopy and LAMP test, respectively. Submicroscopic Plasmodium infection was found in $22.3 \%$ of the children. The independent risk factors for anemia are Plasmodium infection, children younger than 24 months, low middle-upper arm circumference, and history of illness two weeks before. Otherwise, children with submicroscopic malaria infection have a significantly increased risk for anemia, with a need of transfusion. The prevalence of malaria infection was underestimated, when microscopy was used to diagnose malaria. Children with low parasitemia detected by LAMP but not by microscopy showed a significantly increased prevalence of anemia.
\end{abstract}

\section{INTRODUCTION}

In 2018, there were an estimated 228 million new malaria cases worldwide. Most cases were from sub-Saharan Africa (93\%). ${ }^{1}$ Each year, approximately 405,000 deaths are due to malaria. ${ }^{1}$ Children younger than 5 years are particularly susceptible to malaria, with an average of one child dying every 2 minutes. ${ }^{1,2}$ The Democratic Republic of Congo (DRC) is at present one of the most affected countries. ${ }^{1}$ In the DRC, according to the recent Demographic and Health Survey organized in 2014, the prevalence of malaria was $23 \%$ in children younger than 5 years. In South Kivu (SK), this prevalence has been estimated to be $10 \% .{ }^{3}$ Plasmodium falciparum is the most prevalent malarial parasite in sub-Saharan Africa, accounting for $99.7 \%$ of estimated malaria cases in $2018 .{ }^{1}$ It is estimated that anemia affects a quarter of the global population, including 293 million (47\%) children younger than 5 years. ${ }^{4-6}$ In the DRC, $47 \%$ of children aged between 6 and 59 months are affected by anemia: $20 \%$ showing a mild form, $25 \%$ a moderate form, and $2 \%$ a severe form. In SK, this prevalence has been estimated to be $27 \% .{ }^{3}$ Malaria and anemia remain public health problems, with high priority in the DRC, in SK in particular. It has been described that $P$. falciparum causes acute and chronic anemia in endemic areas. ${ }^{7}$ The study carried out by Menendez et al. ${ }^{8}$ in a high malaria transmission zone confirmed the role of Plasmodium in the etiology of anemia in children. Plasmodium falciparum may account for about $60 \%$ of all episodes of severe anemia in children. ${ }^{8}$ Other authors demonstrated the contribution of malaria in the overall prevalence of anemia in young children..$^{9-13}$ The pathophysiological mechanisms of the emergence of anemia in malaria infection has been extensively

* Address correspondence to Jan Philippe, Department Laboratory Medicine, C. Heymanslaan 10, Ghent 9000, Belgium. E-mail: jan.philippe@ uzgent.be studied. Briefly, there is an increased hemolysis of infected and uninfected red cells, inadequate erythropoiesis because of the high level of tumor necrosis factor and macrophage inhibitory factor production, intramedullary deposition of hemozoin, and low levels of interleukin-10. ${ }^{14,15}$ The degree of malarial anemia depends on various factors, among properties of both hosts and parasites. ${ }^{16}$ Immunity is the factor that most strongly determines whether a malaria infection produces symptoms. An individual's level of immunity to infection is determined by past exposure history and age. Increased immunity leads to improved control over parasite multiplication and decreased parasite density, which in turn lessens the severity of symptoms. ${ }^{17}$ In younger children, especially in children younger than 6 months, the presence of maternal antibodies may decrease the prevalence of Plasmodium infection and therefore of asymptomatic infection. Older children (aged $>6$ months), when the maternal protection decreases, are more prone to develop symptomatic or even severe symptomatic malaria episodes resulting in anemia. ${ }^{18-21}$ Chronic subclinical infections tend to be associated with substantially lower parasite densities in contrast with acute symptomatic infections. These chronic infections may be "submicroscopic" and thus are not detected in a blood film or by rapid diagnostic tests (RDTs). Analytically more sensitive methods such as molecular analyses with polymerase chain reaction (PCR) are needed to detect the low parasitemia which can persist for months or years. ${ }^{22}$ In regions of high transmission, children eventually acquire the ability to maintain a parasite density below the level that causes fever, but chronic or repeated infection may cause a state of chronic anemia. ${ }^{15}$ Advances in molecular diagnostic techniques have revealed a larger reservoir of asymptomatic human malarial infections than previously recognized. Several studies have shown the relevance of molecular analysis to detect Plasmodium in asymptomatic children with low parasite density with less than five parasites $/ \mu \mathrm{L}$, not detectable with RDT or 
blood film. ${ }^{23-30}$ This study is the first in this area using an ultrasensitive test in the mass screening to assess a role of a subclinical and submicroscopic Plasmodium infection in the etiology of anemia in children younger than 5 years. The main objective of this study was to explore the prevalence and relationship of malaria and anemia in children younger than 5 years, and more specifically, to investigate the relationship between submicroscopic Plasmodium infection and anemia in children living in the rural area of SK, DRC.

\section{MATERIALS AND METHODS}

Study location. The study was carried out in the health zone $(\mathrm{HZ})$ of Miti Murhesa in the SK Province/DRC. South Kivu is situated in the eastern DRC and shares borders with Rwanda, Burundi, and Tanzania. Bukavu is the capital of the province. The landscape includes mountains (eastern north part), the central basin (western part), and the vast plains in the southern east part. We can distinguish a rainy season from September to May and a dry season from June to August. The province has $34 \mathrm{HZs}$. Miti Murhesa is a rural $\mathrm{HZ}$ located at $30 \mathrm{~km}$ north of Bukavu, situated between 1,500 and 2,000 m of altitude. However, because Miti Murhesa is situated at 1,500-2,000 km of altitude, the period of transmission may be shorter as this area may be considered as a mountain ecosystem. ${ }^{31,32}$

Study design. In this cross-sectional survey, we recruited 1,088 children using a systematic sampling method to select clusters (villages) in the $\mathrm{HZ}$ of Miti Murhesa. Data collection took place from April 5 to May 9, 2018 (end of the rainy season).

Sample size. The sample size for this study was based on a proportion in a single cross-sectional survey, and the estimate of the proportion was based on the prevalence of malaria in SK, which was estimated to be $10 \% .^{3}$ The absolute precision was set at $2.5 \%$, with $95 \% \mathrm{Cl}$, and the design effect of 2 with $5 \%$ of missing. The sample size required for this study was found to be 1,161 children aged 6-59 months. In practice, we opted for 30 clusters (villages) as previously described, ${ }^{33}$ with 38 observations per cluster (30 clusters $\times 38$ observations). Finally, 1,088 children were selected from 30 villages.

Sampling method. A two-stage sampling process was used to determine the study participants. First, we randomly selected 30 villages using the systematic sampling method. The village chief informed us about the number of households. We divided the total number of households by 38 (the number of children to be included per village) to calculate the sampling interval. The first selected household was randomly selected between one and the sampling interval. Then, we continued based on the sampling interval. We included one child, aged 6-59 months, per household who was a permanent resident of Miti Murhesa and whose parents/guardians granted consent for study inclusion and blood sample collection. In households with more than one eligible child, the child was randomly selected. If there was no eligible child in a selected household, the household next was then chosen.

Subject selection. A total of 1,088 children were recruited for the survey. The inclusion criteria were based on age (younger than 5 years), willingness of the parent/guardian of the child to participate in the study demonstrated by completion and signing/thumb printing of the consent form and willingness to provide samples required for the laboratory test, and general good health. We excluded all children with acute illness by measuring temperature $\left(T \geq 37.5^{\circ} \mathrm{C}\right)$, children with significant morbidity (congenital heart disease, severe malformations, and cancer), children with psychomotor retardation, or severely sick children (with diarrhea, vomiting with dehydration, convulsion, and dyspnea); these children were referred to the health center for care.

Study questionnaire. A questionnaire was administered to the child's parent/guardian to collect data on demographic characteristics (gender, age, education level of the head of the family, occupation, and marital status), socioeconomic statusrelated variables (number of house occupants, house type, toilet type, and water source), preventive intervention (history of complementary food before 6 months, exclusive breastfeeding during 6 months, and use of insecticide-treated bed net (ITN) during the night before the survey), and morbidity (fever in the last 2 weeks and antecedent of transfusion).

Clinical evaluation and anthropometric measurements. The axillary temperature was measured using a digital thermometer, and fever was defined as temperature $\geq 37.5^{\circ} \mathrm{C}$; pitting bilateral edema was noticed if present. Experienced healthcare personnel assessed the children's growth by measuring height (children aged $>2$ years) or length (children aged $\leq 2$ years), middle upper arm circumference (MUAC), and weight. Height/length and weight were measured nearest $0.1 \mathrm{~cm}$ and $0.05 \mathrm{~kg}$, respectively. Duplicate measurements were taken using standardized methods and calibrated equipment, ${ }^{34}$ and a third measurement was obtained if the difference between the first two measurements was greater than the allowable difference for that measure. Weight-for-age, height-for-age, and weight-for-height were expressed in Z-scores and calculated with the WHO Anthro (WHO Anthro Geneva version 3.2.2) for Stata software version 13.1 for Mac (StatCorp, College station, TX). Extreme outliers were excluded as follows: weight-for-age $z$-score $(W A Z) \leq-6$ or $\geq 5$, height-for-age $z$-score $(H A Z) \leq-6$ or $\geq 5$, and weight-for-height $z$-score $(W H Z) \leq-6$ or $\geq 5$. A child was identified as being malnourished if he or she scored $<-2 S D$ for one of the anthropometric indices: HAZ (stunting), WAZ (underweight), and $\mathrm{WHZ}$ (wasting). If the corresponding indices were scored $<-3 \mathrm{SD}$, the child was considered having severe undernutrition.

Blood collection and laboratory tests. Hemoglobin $(\mathrm{Hb})$ was determined by finger prick by the Hemocue $301+$ instrument (Angelhom, Sweden). The WHO criteria were used to define and classify anemia in children aged 6-59 months. ${ }^{35}$ Anemia was defined as $\mathrm{Hb}<11 \mathrm{~g} / \mathrm{dL}$ and classified into mild if $\mathrm{Hb}$ was in the range 10-10.9 g/dL, moderate if $\mathrm{Hb}$ was $7-9.9 \mathrm{~g} /$ $\mathrm{dL}$, and severe if $\mathrm{Hb}$ was $<7 \mathrm{~g} / \mathrm{dL}$. Hemoglobin was adjusted for altitude as proposed by Sullivan KM et al. ${ }^{36} \mathrm{~A}$ blood sample was collected by venipuncture into two tubes, one with ethylenediaminetetraacetic acid (EDTA) $(4 \mathrm{~mL})$ and one without anticoagulant $(4 \mathrm{~mL})$. The tubes were immediately stored in cooler boxes containing ice packs and were transported to the field laboratory of the Provincial Reference General Hospital of Bukavu for malaria microscopy and loop-mediated isothermal amplification (LAMP)-based illumigene malaria assay analysis. Slides were stained with $10 \%$ Giemsa and read by two independent, trained, and experienced microscopists. If necessary, a third independent microscopist was added to resolve discrepant results. A slide was considered negative after 200 microscopic fields were examined in the thick smear. The LAMP assay was performed using the illumipro- $10^{\mathrm{TM}}$ incubator/reader, which is capable of testing 10 samples per run. The illumigene malaria amplification assay was used. 
The illumigene assay is a qualitative in vitro diagnostic LAMP test for the detection of Plasmodium spp. DNA in human venous EDTA whole blood samples. The assay targets a region of the Plasmodium genome that is conserved across $P$. falciparum, vivax, ovale, malariae, and knowlesi. ${ }^{37}$ The assay does not distinguish between the different Plasmodium species. The assay uses a simple filtration workflow $\left(S M P-P R E P^{T M}\right)$ to extract DNA from EDTA anticoagulated whole blood, a procedure relying on chemical lysis which produces amplifiable DNA within 10 minutes. ${ }^{37,38}$ The change in turbidity associated with LAMP assays, due to the magnesium-pyrophosphate build up as a by-product, is measured by the illumipro-10 reader, and a qualitative result is determined.

We defined an infection as asymptomatic if Plasmodium parasites without fever were identified. Submicroscopic Plasmodium parasitemia was defined as a negative thick smear with a positive LAMP illumigene malaria assay. To define the group of submicroscopic Plasmodium parasitemia, we excluded all children who had concurrently positive thick smear and LAMP test and selected those with exclusively LAMP-positive tests.

Statistical analysis. Data were double-entered in Microsoft Excel (Microsoft 2016, Santa Rosa, CA), and analyses were performed using MedCalc ${ }^{\circledR}$ software version 9.4.2.0 (MedCalc, Mariakerke, Belgium). Continuous variables ( $\mathrm{Hb}$ and age) were expressed as median \pm interquartile range or mean $\pm S D$, and categorical variables were summarized by proportions. The chi square test was used to compare proportions.

A multivariate logistic regression model, based on forward stepwise analysis, was used to determine adjusted odds for risk associated with anemia and submicroscopic Plasmodium infection.

TABLE 1

Baseline characteristics of the study population and its association with anemia in children

\begin{tabular}{|c|c|c|c|}
\hline Variable & $N(\%)$ & Number of children with anemia, $n(\%=n / N)$ & $P$-value \\
\hline Age-group (months) & $1,088(100)$ & $431(39.6)$ & \\
\hline $0-23$ & 368 (33.8) & $176(47.8)$ & $<0.0001$ \\
\hline 24-59 & $720(66.2)$ & $255(35.4)$ & \\
\hline Gender & $1,088(100)$ & 431 (39.6) & \\
\hline Male & $545(50.1)$ & $232(42.6)$ & 0.05 \\
\hline Female & 543 (49.9) & $199(36.6)$ & \\
\hline Education level of the head of the household & $1,070(100)$ & $423(39.5)$ & \\
\hline Illiterate & $350(32.7)$ & $142(40.6)$ & \\
\hline Primary & 366 (34.2) & $130(35.5)$ & \\
\hline Secondary and over & $354(33.1)$ & $151(42.7)$ & 0.13 \\
\hline Household size & $1,088(100)$ & 431 (39.6) & \\
\hline $1-5$ & $366(33.7)$ & $152(41.5)$ & 0.002 \\
\hline $6-10$ & $627(57.6)$ & $257(41.0)$ & \\
\hline$>10$ & $95(8.7)$ & $22(23.2)$ & \\
\hline $\begin{array}{l}\text { History of complementary food before } 6 \\
\text { months of age }\end{array}$ & $1,088(100)$ & $431(39.6)$ & \\
\hline Yes & $506(46.5)$ & $194(38.3)$ & 0.45 \\
\hline No & $582(53.5)$ & $237(40.7)$ & \\
\hline Use of insecticide-treated bed net & $1,088(100)$ & 431 (39.6) & \\
\hline Yes & $675(62.0)$ & $264(39.1)$ & 0.71 \\
\hline No & $413(38.0)$ & $167(40.4)$ & \\
\hline History of transfusion & $1,088(100)$ & 431 (39.6) & \\
\hline Yes & $205(18.8)$ & $93(45.4)$ & 0.07 \\
\hline No & $883(81.2)$ & 338 (38.3) & \\
\hline History of illness two weeks before & $1,088(100)$ & 431 (39.6) & \\
\hline Yes & $742(68.2)$ & 326 (43.9) & $<0.0001$ \\
\hline No & $346(31.8)$ & $105(30.3)$ & \\
\hline Edema & $1,088(100)$ & 431 (39.6) & \\
\hline Yes & $45(4.1)$ & $16(35.6)$ & 0.67 \\
\hline No & $1,043(95.9)$ & 415 (39.8) & \\
\hline Middle upper arm circumference (mm) & $1,088(100)$ & $431(39.6)$ & \\
\hline$<125$ & $210(19.3)$ & $123(58.6)$ & $<0.0001$ \\
\hline$\geq 125$ & $878(80.7)$ & $308(35.1)$ & \\
\hline WHZ & $1,069(100)$ & $420(39.3)$ & \\
\hline$<-2$ & $93(8.7)$ & $53(57.0)$ & 0.0004 \\
\hline$\geq-2$ & $976(91.3)$ & 367 (37.6) & \\
\hline HAZ & $1,055(100)$ & 415 (39.3) & \\
\hline$<-2$ & $631(59.8)$ & $251(39.8)$ & 0.76 \\
\hline$\geq-2$ & $424(40.2)$ & $164(38.9)$ & \\
\hline WAZ & $1,083(100)$ & 429 (39.6) & \\
\hline$<-2$ & $367(33.9)$ & $161(43.9)$ & 0.04 \\
\hline$\geq-2$ & $716(66.1)$ & $268(37.4)$ & \\
\hline Thick smear & $1,088(100)$ & 431 (39.6) & \\
\hline Positive & $173(15.9)$ & $119(68.8)$ & $<0.0001$ \\
\hline Negative & $915(84.1)$ & $312(34.1)$ & \\
\hline Loop-mediated isothermal amplification test & $1,057(100)$ & $424(40.1)$ & \\
\hline Positive & $359(34.0)$ & $218(60.7)$ & $<0.0001$ \\
\hline Negative & $698(66.0)$ & 206 (29.5) & \\
\hline
\end{tabular}


Ethical consideration. Ethical approval was obtained by the Institutional Ethical Committee of the Catholic University of Bukavu, UCB (Ref: UCB/CIE/NC/003/2017). Written informed consent was collected from the parents/guardians for all children before inclusion. All children with fever $(T \geq 37.5)$, $\mathrm{Hb}<10 \mathrm{~g} / \mathrm{dL}$, and MUAC $<125 \mathrm{~mm}$ were referred to the health center for care.

\section{RESULTS}

Baseline characteristics of study population. The baseline characteristics of the study population and their association with anemia are summarized in Table 1. A total of 1,088 children aged 6-59 months participated in this study, of whom 431 (39.6\%) were anemic; $66.2 \%$ of children were aged between 24 and 59 months. Anemia was more prevalent among children younger than 24 months $(P<0.001)$. The proportion of boys was $50.1 \%$, and anemia was more prevalent in males (42.6\%) than in females (36.6\%) $(P=0.05)$. No difference was observed in the education level of the head of the household, comparing anemic versus non-anemic children. The majority $(57.6 \%)$ of children live in households with a people size of $6-10$, but anemia was less prevalent in children from the household sizes exceeding 10 people $(P=$ 0.002). There was no difference related to the addition of complementary food. The majority of children (62\%) slept under ITN. No difference was observed between anemic and non-anemic children; $18.8 \%$ of children reported having received a transfusion in the past. In anemic children, a history of transfusion was more frequent than in non-anemic children, but the difference between the two groups was not significant. The majority of children $(68.2 \%)$ reported an antecedent of illness during the 2 weeks preceding the survey. This was more frequent in anemic children than in nonanemic children $(P<0.0001)$. Bilateral pitting edema was observed in $4.1 \%$, and edema was higher among nonanemic children (64.4\%), but no association was observed. The prevalence of MUAC $<125 \mathrm{~mm}$, wasting $(\mathrm{WHZ}<-2)$, stunting $(\mathrm{HAZ}<-2)$, and underweight $(W A Z<-2)$ were seen, respectively, in $19.3 \%, 8.7 \%, 59.8 \%$, and $33.9 \%$. Middle upper arm circumference < $125 \mathrm{~mm}$, wasting, and underweight were significantly associated with anemia. For the LAMP malaria test, 31 results were not available, and 11 results were invalid, and in 20 children, an insufficient amount of blood was collected to perform the analysis. Overall, in $15.9 \%$ children, a positive thick smear was found. Infected children had an increased chance to be anemic compared with noninfected children $(P<0.0001)$; $34.0 \%$ children had a positive LAMP test. Asymptomatic Plasmodium parasitemia was associated with anemia $(P<0.0001)$.

A Multiple logistic regression model for factors associated with anemia in children from Miti Murhesa, SK. In a multiple logistic regression model (Table 2), asymptomatic Plasmodium infection with positive thick smear and LAMP test increase the risk for anemia in a highly significant way with odds ratios (ORs) (2.59; 95\% Cl: 1.63-4.10 and 2.87; 95\% Cl: 2.04-4.01), respectively. Besides, age less than 24 months, history of illness 2 weeks before, and low MUAC increased the risk for anemia with OR, respectively, 2.17, 1.70, and 2.63. However, children living in the household with a size of more than 10 showed a decreased risk for anemia (OR $=0.78 ; 95 \%$ $\mathrm{Cl}$ : 0.62-0.99).
TABLE 2

Multiple logistic regression model for factors associated with anemia in children

\begin{tabular}{|c|c|c|}
\hline Variable & aOR (95\% Cl) & $P$-value \\
\hline \multicolumn{3}{|c|}{ Age-group (months) } \\
\hline 6-23 & $2.17(1.62-2.91)$ & $<0.0001$ \\
\hline $24-59$ & 1 & \\
\hline \multicolumn{3}{|c|}{ Household size } \\
\hline $1-10$ & 1 & 0.04 \\
\hline$>10$ & $0.78(0.62-0.99)$ & \\
\hline \multicolumn{3}{|c|}{ History of illness 2 weeks before } \\
\hline Yes & $1.70(1.25-2.29)$ & 0.0005 \\
\hline No & 1 & \\
\hline \multicolumn{3}{|c|}{ Middle upper arm circumference (mm) } \\
\hline$<125$ & $2.63(1.88-3.69)$ & $<0.0001$ \\
\hline$\geq 125$ & 1 & \\
\hline \multicolumn{3}{|l|}{ Thick smear } \\
\hline Positive & $2.59(1.63-4.10)$ & $<0.0001$ \\
\hline Negative & 1 & \\
\hline \multicolumn{3}{|c|}{ Loop-mediated isothermal amplification malaria test } \\
\hline Positive & $2.87(2.04-4.01)$ & $<0.0001$ \\
\hline Negative & 1 & \\
\hline
\end{tabular}

Submicroscopic Plasmodium infection. Table 3 summarizes the demographic characteristics of the study population and its association with submicroscopic Plasmodium infection. The prevalence of submicroscopic Plasmodium infection was found to be $22.3 \%$. Submicroscopic Plasmodium infection was higher in children aged 24 months and older $(P=$ 0.02). Asymptomatic submicroscopic Plasmodium infection was higher in boys than in girls $(P=0.004)$. Children who did not receive complementary feeding before 6 months were more prone to have submicroscopic Plasmodium infection $(P=0.01)$. A history of transfusion was associated with submicroscopic Plasmodium infection with $P=0.005$. Anemia was significantly associated with submicroscopic Plasmodium infection $(P<0.0001)$.

In the multiple logistic regression model (Table 4), the risk factors predicting submicroscopic Plasmodium infection included anemia, age, male gender, and a history of transfusion. The absence of extra feeding before 6 months of age was also associated with submicroscopic Plasmodium infection.

Anemia increases the finding of submicroscopic Plasmodium infection by 2.75 (95\% Cl: $1.96-3.85, P<0.0001)$. Otherwise, children younger than 24 months were less at risk for submicroscopic Plasmodium infection $(\mathrm{OR}=0.56$; $95 \% \mathrm{Cl}$ : $0.39-0.80, P=0.001)$. Finally, a history of transfusion in the past and male gender predict a submicroscopic Plasmodium infection $(\mathrm{OR}=1.75$; $95 \% \mathrm{Cl}$ : $1.16-2.63, P=0.007)(\mathrm{OR}=1.59$; $95 \% \mathrm{Cl}: 1.15-2.22, P=0.005)$, respectively, whereas the extra feeding before 6 months shows a decreased risk for submicroscopic Plasmodium infection $(\mathrm{OR}=0.64 ; 95 \% \mathrm{Cl}$ : $0.46-0.90, P=0.01)$.

\section{DISCUSSION}

In this study, we examined the prevalence of anemia and focused on the relationship of submicroscopic Plasmodium infection and anemia in children younger than 5 years living in the rural area of SK/DRC. We found that anemia was most prevalent in children younger than 2 years. By contrast, submicroscopic Plasmodium infection was most prevalent in older children (aged 3-5 years). Nevertheless, anemia and submicroscopic Plasmodium infection strongly correlated 
TABLE 3

Baseline characteristics of study population and its association with submicroscopic Plasmodium infection (PI)

\begin{tabular}{|c|c|c|c|}
\hline Variable & $N(\%)$ & Submicroscopic PI, $n(\%=n / M)$ & $P$-value \\
\hline Age-group (months) & $898(100)$ & $200(22.3)$ & \\
\hline $6-23$ & 326 (36.3) & $59(18.0)$ & 0.02 \\
\hline $24-59$ & $572(63.7)$ & $141(24.7)$ & \\
\hline Gender & $898(100)$ & $200(22.3)$ & \\
\hline Male & $434(48.3)$ & $115(26.5)$ & 0.004 \\
\hline Female & 464 (51.7) & 85 (18.3) & \\
\hline Education level of the head of household & $882(100)$ & $197(22.3)$ & \\
\hline Illiterate & $291(33.0)$ & $54(18.6)$ & 0.15 \\
\hline Primary & $295(33.4)$ & $73(24.7)$ & \\
\hline Secondary and over & 296 (33.6) & $70(23.6)$ & \\
\hline Household size & $898(100)$ & $200(22.3)$ & \\
\hline $1-5$ & 305 (34.0) & $68(22.3)$ & 0.54 \\
\hline $6-10$ & 508 (56.6) & $117(23.0)$ & \\
\hline$>10$ & $85(9.4)$ & $15(17.6)$ & \\
\hline $\begin{array}{l}\text { History of complementary food before } 6 \\
\text { months of age }\end{array}$ & $989(100)$ & $200(22.3)$ & \\
\hline Yes & $421(46.9)$ & $78(18.5)$ & \\
\hline No & $477(53.1)$ & $122(25.6)$ & 0.01 \\
\hline Use of insecticide-treated bed net & $898(100)$ & $200(22.3)$ & \\
\hline Yes & $563(62.7)$ & $120(21.3)$ & 0.41 \\
\hline No & 335 (37.3) & $80(23.9)$ & \\
\hline History of transfusion & $898(100)$ & $200(22.3)$ & \\
\hline Yes & $147(16.4)$ & $46(31.3)$ & 0.005 \\
\hline No & 751 (83.6) & $154(20.5)$ & \\
\hline History of illness 2 weeks before & $898(100)$ & $200(22.3)$ & \\
\hline Yes & 604 (67.3) & $140(23.2)$ & 0.39 \\
\hline No & $294(32.7)$ & $60(20.4)$ & \\
\hline Edema & $898(100)$ & $200(22.3)$ & \\
\hline Yes & $36(4.0)$ & $5(13.9)$ & 0.30 \\
\hline No & $862(96.0)$ & $195(21.7)$ & \\
\hline Middle upper arm circumference (mm) & $898(100)$ & $200(22.3)$ & \\
\hline$<125$ & $167(18.6)$ & $39(23.4)$ & 0.78 \\
\hline$\geq 125$ & $731(81.4)$ & $161(22.0)$ & \\
\hline WHZ & $885(100)$ & $198(22.4)$ & \\
\hline$<-2$ & $72(8.1)$ & $16(22.2)$ & 0.90 \\
\hline$\geq-2$ & $813(91.9)$ & $182(22.4)$ & \\
\hline HAZ & $869(100)$ & $190(21.9)$ & \\
\hline$<-2$ & $517(59.5)$ & $105(20.3)$ & 0.20 \\
\hline$\geq-2$ & $352(40.5)$ & $85(24.1)$ & \\
\hline WĀZ & $895(100)$ & $200(22.3)$ & \\
\hline$<-2$ & 301 (33.6) & $64(21.3)$ & 0.63 \\
\hline$\geq-2$ & $594(66.4)$ & $136(22.9)$ & \\
\hline Anemia & $898(100)$ & $200(22.3)$ & \\
\hline Yes & 309 (34.4) & 103 (33.3) & $<0.0001$ \\
\hline No & $589(65.6)$ & $97(16.5)$ & \\
\hline
\end{tabular}

pointing to a prominent role of the submicroscopic malaria infection in anemia, particularly in the age-group of 3-5 years. In general, almost $40 \%$ of children were anemic. This prevalence is in agreement with data previously described in the region. Two independent studies described the prevalence of anemia in the same region up to $36 \%{ }^{3}$ and $46.6 \% .^{39} \mathrm{~A}$ third study found the prevalence of $58.6 \%$ and $35.4 \%$ in the agegroups 6-23 months and $24-59$ months, ${ }^{40}$ respectively. In a demographic investigation performed in $2007,{ }^{41}$ anemia was found up to $60 \%$, decreasing towards $36 \%$ in 2013. Our present data confirm that anemia remains a major health problem for the SK region. Regarding the prevalence of Plasmodium infection, surprisingly, in our study, a marked different score was found when compared with previous results. Our study shows a higher prevalence of malaria up to $16 \%$ and $34 \%$ using thick smear and LAMP malaria test, respectively. This is far above the scores found in previous studies performed in the same region. ${ }^{3,39}$ In 2014, a survey described the prevalence of malaria in SK of $12 \%$ and $10 \%$ with RDTs and thick smear, respectively. Differences may be due to the sensitivity of the tests used in the two surveys, and to seasonal differences. The LAMP test is more sensitive, but thick smear examinations also showed a higher prevalence than was previously reported. The same survey in 2014 showed $10.4 \%$ positive cases when PCR was applied. ${ }^{42}$ This is surprisingly not higher than the findings by microscopy and RDT, and was also far below the numbers we found with the LAMP assay. Our study was performed at the end of the rainy season, associated with higher malaria transmission. A temporal change in transmission associated with climate change could also explain in part a rise in the prevalence of malaria. ${ }^{31,32}$ One more reason could be an increased mosquito resistance to insecticides. We did not find any difference in the prevalence of parasitemia between children who used or did not use ITN. We should take into consideration that observed results might be explained by an inferior quality of the ITN because of a too long storage time. Four other studies, at least, did find a protective effect of ITN. ${ }^{43-46}$ However, Gitonga et al. ${ }^{44}$ in Kenya described that in 
TABLE 4

Multiple logistic regression model for factors associated with submicroscopic Plasmodium infection in children

\begin{tabular}{lcc}
\hline Variable & aOR & $P$-value \\
\hline $\begin{array}{l}\text { Age-group (months) } \\
6-23\end{array}$ & $0.56(0.39-0.80)$ & \\
$24-59$ & 1 & 0.001 \\
Gender & & \\
$\quad$ Male & $1.59(1.15-2.22)$ & 0.005 \\
$\quad$ Female & 1 & \\
History of complementary food before 6 months of age & \\
$\quad$ Yes & $0.64(0.46-0.90)$ & 0.01 \\
$\quad$ No & 1 & \\
History of transfusion & $1.75(1.16-2.63)$ & 0.007 \\
$\quad$ Yes & 1 & \\
No & & \\
Anemia & & \\
Yes & 1 & \\
No & $2.75(1.96-3.85)$ & \\
\hline
\end{tabular}

some zones, the protective effect of ITN could not be confirmed. Recently, a study conducted in North Ubangi/DRC has demonstrated resistance of Anopheles gambiae to deltamethrin, permethrin, and dichlorodiphenyltrichloroethane. ${ }^{47}$ Mosquito resistance to insecticides has been described elsewhere in the DRC. ${ }^{48}$ Other factors playing a role in the rise of malaria are resistant to antimalarial drugs, the climate change, and the migration of people in the context of civil war. ${ }^{32,49-52}$

Risk factors for anemia. A forward stepwise multiple regression analysis was used to identify the factors predicting anemia. Age, household size less than 10, history of illness 2 weeks before, MUAC $<125 \mathrm{~mm}$, and Plasmodium infection remained significant for prediction of anemia. Children younger than 24 months were at increased risk of anemia, which is in agreement with previous results. ${ }^{39,40}$ This can be due to the fact that many other causes of anemia play an important role such as increased requirement of iron in this period of rapid child growth, inadequate iron intake, and repeated infections. Malnourished children are at increased risk of micronutrient deficiencies and are more likely to suffer from impaired immune function with more infections, also parasitic infections. $^{6,21,53}$ These considerations may explain that children who reported a history of illness in the previous 2 weeks are more prone to anemia. The pathophysiology of anemia during an acute phase of infection or inflammation is not clearly established, but studies have demonstrated that children with acute infection experience a significant drop in $\mathrm{Hb}^{.54-56}$ Importantly, we found a clear relationship between asymptomatic Plasmodium infection and anemia. Our findings are consistent with previous studies in sub-Saharan Africa concerning anemia. ${ }^{21,44,45,57,58}$ Other findings, such as the correlation of weight and MUAC, are expected. In the logistic regression analysis, weight disappears and only MUAC remains, indicating that MUAC is the most important one. To our surprise, the biggest households showed the lowest anemia prevalence: one idea is that socioeconomic status may play a role.

Submicroscopic Plasmodium infection and risk factors. Multiple logistic regression analyses showed that independent variables predicting asymptomatic submicroscopic Plasmodium infection were age, anemia, gender, absence of complementary feeding before 6 months, and history of transfusion. Increasing age predicted submicroscopic Plasmodium infection. Our finding was consistent with other studies in Africa. ${ }^{58,59}$ Increasing age has been clearly linked to lower parasite densities. In older children, more infection tend to be submicroscopic; this is probably because of a greater immunity in older children induced by cumulative exposure and developed immunity than in younger children who are more prone to develop symptomatic malaria. ${ }^{17,19,20,59,60}$ In our study, we found a relationship between submicroscopic Plasmodium infection and anemia. Children with anemia were 2.7 times more prone (95\% Cl: $1.96-3.85)$ to have a submicroscopic Plasmodium infection. This finding is consistent with the study in Rwanda, where $28 \%$ of children with submicroscopic Plasmodium infection were anemic. ${ }^{29}$ In general, asymptomatic, especially submicroscopic Plasmodium infection, has received less attention than symptomatic malaria. Submicroscopic carriers were found to be a source of human to mosquito transmission. ${ }^{59,61}$ A history of transfusion also predicted submicroscopic Plasmodium infection. This illustrates that children at younger age may have overt malaria, needing transfusion, and remain asymptomatic carriers in the long term. Our study shows that children receiving exclusive breastfeeding during the first 6 months of life are at increased risk of submicroscopic Plasmodium infection. These results are in contrast with the studies conducted in Kinshasa/DRC ${ }^{62}$ and in Malawi, ${ }^{63}$ in which exclusive breastfeeding was associated with reduced risk of malaria infection in young children, whereas in Uganda, the protective effect of exclusive breastfeeding was not demonstrated. ${ }^{64}$ The WHO recommends exclusive breastfeeding for 6 months. Breastfeeding brings clear benefits for child health by reducing morbidity and mortality from infectious diseases. ${ }^{65}$

\section{CONCLUSION}

The strong association between malaria and anemia is well known. This is also true in the SK region of the DRC. Importantly, anemia is also strongly associated with asymptomatic submicroscopic malaria, especially in malnourished children aged younger than 3 years and who were ill shortly before. The submicroscopic infection is most relevant in boys in the agegroup of 24-59 months. In our study, the use of ITN did not offer a clear protection against malaria. This is a controversial finding, needing further exploration. It may be recommended to offer preventive malaria treatment in this age-group, and this is even more meaningful if the child is anemic, even in the absence of clinical malaria symptoms or negative thick smears. Because this study is cross-sectional and not longitudinal, we cannot make any predictions on the clinical outcome.

Received November 28, 2019. Accepted for publication January 18, 2020.

Published online March 2, 2020.

Acknowledgments: We thank the staff of the laboratory of the General Provincial Reference Hospital of Bukavu, and also the field-workers. We thank all the study participants for agreeing to participate in this study. Sander De Bruyne is thanked for sharing his expertise in statistical analysis.

Financial support: This work was supported by the Vlaamse Interuniversitaire Raad-Universitaire Ontwikkelings samenwerking Cooperation (VLIR-UOS) (grant number CD201IUC024A101).

Disclosure: Ethics approval and informed consent to participate were obtained from all subjects.

Authors' addresses: Yvette Lufungulo Bahati, Department of Pediatrics, Catholic University of Bukavu, Bukavu, Democratic Republic of Congo, and Department of Diagnostic Sciences, Ghent University, Ghent, Belgium, E-mail: yvebahati@gmail.com. Joris delanghe and Jan Philippé, Department of Diagnostic Sciences, Ghent University, 
Ghent, Belgium, E-mails: yvebahati@gmail.com and jan.philippe@ ugent.be. Ghislain Bisimwa Balaluka, Department of Public Health, Catholic University of Bukavu, Bukavu, Democratic Republic of Congo, E-mail: ghislainbiba@yahoo.fr. Antoine Sadiki Kishabongo, Department of Clinical Biology, Catholic University of Bukavu, Bukavu, Democratic Republic of Congo, E-mail: santoines@yahoo.fr.

\section{REFERENCES}

1. World Health Organization, 2019. World Malaria Report 2019. Geneva, Switzerland: WHO.

2. World Health Organization, 2016. World Malaria Report 2016. Geneva, Switzerland: WHO.

3. The Ministry of Monitoring PalotMR, The Ministry of Health Public, ICF Intemational, 2014. Demographic and Health Sunvey 2013-2014. Rockville, MD, MPSMRM, MSP \& ICF Intemational.

4. World Health Organization, 2015. The Global Prevalence of Anaemia in 2011. Geneva, Switzerland: WHO.

5. Zuffo CR, Osorio MM, Taconeli CA, Schmidt ST, da Silva BH, Almeida CC, 2016. Prevalence and risk factors of anemia in children. J Pediatr 92: 353-360.

6. Balarajan Y, Ramakrishnan U, Özaltin E, Shankar AH, Subramanian SV, 2011. Anaemia in low-income and middle-income countries. Lancet 378: 2123-2135.

7. Makani J, Roberts DJ, 2016. Hematology in Africa. Hematol Oncol Clin North Am 30: 457-475.

8. Menendez C et al., 1997. Randomised placebo-controlled trial of iron supplementation and malaria chemoprophylaxis for prevention of severe anaemia and malaria in Tanzanian infants. Lancet 350: 844-850.

9. White NJ, 2018. Anaemia and malaria. Malar J 17: 371.

10. Korenromp EL, Armstrong-Schellenberg JR, Williams BG, Nahlen BL, Snow RW, 2004. Impact of malaria control on childhood anaemia in Africa a quantitative review. Trop Med Int Health 9: 1050-1065.

11. Hershey CL et al., 2017. Malaria control interventions contributed to declines in malaria parasitemia, severe anemia, and all-cause mortality in children less than 5 years of age in Malawi, 2000-2010. Am J Trop Med Hyg 97 (3 Suppl): 76-88.

12. Ferrari G, Ntuku HM, Ross A, Schmidlin S, Kalemwa DM, Tshefu AK, Lengeler C, 2016. Identifying risk factors for Plasmodium infection and anaemia in Kinshasa, Democratic Republic of Congo. Malar J 15: 362.

13. Kiggundu VL et al., 2013. High prevalence of malaria parasitemia and anemia among hospitalized children in Rakai, Uganda. PLoS One 8: e82455.

14. Pathak VA, Ghosh K, 2016. Erythropoiesis in malaria infections and factors modifying the erythropoietic response. Anemia 2016: 9310905.

15. Roberts DJ, 2016. Hematologic changes associated with specific infections in the tropics. Hematol Oncol Clin North Am 30: 395-415.

16. Phillips RE, Pasvol G, 1992. Anaemia of Plasmodium falciparum malaria. Baillieres Clin Haematol 5: 315-330.

17. Lindblade KA, Steinhardt L, Samuels A, Kachur SP, Slutsker L, 2013. The silent threat: asymptomatic parasitemia and malaria transmission. Expert Rev Anti Infect Ther 11: 623-639.

18. Jagannathan $P$, Muhindo MK, Kakuru A, Arinaitwe E, Greenhouse B, Tappero J, Rosenthal PJ, Kaharuza F, Kamya MR, Dorsey G, 2012. Increasing incidence of malaria in children despite insecticide-treated bed nets and prompt anti-malarial therapy in Tororo, Uganda. Malar J 11: 435.

19. Amaratunga C, Lopera-Mesa TM, Brittain NJ, Cholera R, Arie T, Fujioka H, Keefer JR, Fairhurst RM, 2011. A role for fetal hemoglobin and maternal immune IgG in infant resistance to Plasmodium falciparum malaria. PLoS One 6: e14798.

20. Billig EM, McQueen PG, McKenzie FE, 2012. Foetal haemoglobin and the dynamics of paediatric malaria. Malar J 11: 396.

21. Maketa V, Mavoko HM, da Luz RI, Zanga J, Lubiba J, Kalonji A, Lutumba P, Van Geertruyden JP, 2015. The relationship between Plasmodium infection, anaemia and nutritional status in asymptomatic children aged under five years living in stable transmission zones in Kinshasa, Democratic Republic of Congo. Malar J 14: 83.
22. Chen I, Clarke SE, Gosling R, Hamainza B, Killeen G, Magill A, O'Meara W, Price RN, Riley EM, 2016. "Asymptomatic" malaria: a chronic and debilitating infection that should be treated. PLOS Med 13: e1001942.

23. Elbadry MA et al., 2015. High prevalence of asymptomatic malaria infections: a cross-sectional study in rural areas in six departments in Haiti. Malar J 14: 510.

24. Mvumbi DM, Bobanga TL, Melin P, De Mol P, Kayembe JM, Situakibanza HN, Mvumbi GL, Nsibu CN, Umesumbu SE, Hayette MP, 2016. High prevalence of Plasmodium falciparum infection in asymptomatic individuals from the Democratic Republic of the Congo. Malar Res Treat 2016: 5405802.

25. Carrasco-Escobar G, Miranda-Alban J, Fernandez-Minope C, Brouwer KC, Torres K, Calderon M, Gamboa D, LlanosCuentas A, Vinetz JM, 2017. High prevalence of very-low Plasmodium falciparum and Plasmodium vivax parasitaemia carriers in the Peruvian Amazon: insights into local and occupational mobility-related transmission. Malar J 16: 415.

26. Ataei S, Nateghpour M, Hajjaran H, Edrissian GH, Foroushani AR, 2011. High specificity of semi-nested multiplex PCR using dried blood spots on DNA Banking Card in comparison with frozen liquid blood for detection of Plasmodium falciparum and Plasmodium vivax. J Clin Lab Anal 25: 185-190.

27. Lamptey H, Ofori MF, Kusi KA, Adu B, Owusu-Yeboa E, KyeiBaafour E, Arku AT, Bosomprah S, Alifrangis M, Quakyi IA, 2018. The prevalence of submicroscopic Plasmodium falciparum gametocyte carriage and multiplicity of infection in children, pregnant women and adults in a low malaria transmission area in southern Ghana. Malar J 17: 331.

28. Kavunga-Membo H, llombe G, Masumu J, Matangila J, Imponge J, Manzambi E, Wastenga F, Ngoyi DM, Van Geetruyden JP, Muyembe JJ, 2018. Molecular identification of Plasmodium species in symptomatic children of Democratic Republic of Congo. Malar J 17: 334.

29. Gahutu JB et al., 2011. Prevalence and risk factors of malaria among children in southern highland Rwanda. Malar J 10: 134.

30. Girma S, Cheaveau J, Mohon AN, Marasinghe D, Legese R, Balasingam N, Abera A, Feleke SM, Golassa L, Pillai DR, 2018. Prevalence and epidemiological characteristics of asymptomatic malaria based on ultrasensitive diagnostics: a crosssectional study. Clin Infect Dis 69: 1003-1010.

31. PNLP, KSPH, Swiss KSPH, INRB and INFORM 2014. An Epidemiological Profile of Malaria in the Democratic Republic of Congo. A Report Prepared for the Federal Ministry of Health, Democratic Republic of Congo, the Roll Back Malaria Partnership and the Department for International Development, UK.

32. Archin BS, 2018. Malaria risk factors and intervention policies in Democratic Republic of Congo. Res J Biol 6: 7-13.

33. Gorstein J, Sullivan KM, Parvanta I, Begin F, 2007. Indicators and Methods for Crossectional Surveys of Vitamin and Mineral Status of Populations. Ottawa, ON: The Micronutrient Initiative and Atlanta, GA: The Centers for Disease Control and Prevention, 155.

34. Cogill B, 2003. Anthropometric Indicators Measurement Guide. Washington, DC: Food and Nutrition Technical Assistance (FANTA) Project.

35. World Health Organization, 2011. Haemoglobin Concentrations for the Diagnosis of Anaemia and Assessment of Severity. Vitamin and Mineral Nutrition Information System. Geneva, Switzerland: WHO.

36. Sullivan KM, Mei Z, Grummer-Strawn L, Parvanta I, 2008. Haemoglobin adjustments to define anaemia. Trop Med Int Health 13: $1267-1271$.

37. Lucchi NW et al., 2016. Evaluation of the illumigene malaria LAMP: a robust molecular diagnostic tool for malaria parasites. Sci Rep 6: 36808.

38. De Koninck AS, Cnops L, Hofmans M, Jacobs J, Van den Bossche D, Philippe J, 2017. Diagnostic performance of the loopmediated isothermal amplification (LAMP) based illumigene ${ }^{\circledR}$ malaria assay in a non-endemic region. Malar J 16: 418.

39. Bahizire E, Bahwere $P$, Donnen $P$, Tugirimana PL, Balol'ebwami S, Dramaix M, Nfundiko C, Chirimwami R, Mubagwa K, 2017. High prevalence of anemia but low level of iron deficiency in preschool children during a low transmission period of malaria in 
rural Kivu, Democratic Republic of the Congo. Am J Trop Med Hyg 97: 489-496.

40. Harvey-Leeson S et al., 2016. Anemia and micronutrient status of women of childbearing age and children 6-59 months in the Democratic Republic of the Congo. Nutrients 8: 98.

41. Ministère du Plan et Macro International, 2008. Enquête Démographique et de Santé, République Démocratique du Congo 2007. Calverton, MD: Ministère du Plan et Macro International.

42. The Ministry of Monitoring PalotMR, The Ministry of Health Public, ICF International, 2014. Demographic and Health Survey 2013-2014: Supplemental Malaria Report. Rockville, MD: MPSMRM, MSP, and ICF International.

43. Winskill P, Rowland M, Mtove G, Malima RC, Kirby MJ, 2011. Malaria risk factors in north-east Tanzania. Malar J 10: 98.

44. Gitonga CW, Edwards T, Karanja PN, Noor AM, Snow RW, Brooker SJ, 2012. Plasmodium infection, anaemia and mosquito net use among school children across different settings in Kenya. Trop Med Int Health 17: 858-870.

45. Sultana M, Sheikh N, Mahumud RA, Jahir T, Islam Z, Sarker AR, 2017. Prevalence and associated determinants of malaria parasites among Kenyan children. Trop Med Health 45: 25.

46. Maziarz $\mathrm{M}$ et al., 2018. A cross-sectional study of asymptomatic Plasmodium falciparum infection burden and risk factors in general population children in 12 villages in northern Uganda. Malar J 17: 240.

47. Lynd A, Oruni A, Van't Hof AE, Morgan JC, Naego LB, Pipini D, O'Kines KA, Bobanga TL, Donnelly MJ, Weetman D, 2018. Insecticide resistance in Anopheles gambiae from the northern Democratic Republic of Congo, with extreme knockdown resistance $(\mathrm{kdr})$ mutation frequencies revealed by a new diagnostic assay. Malar J 17: 412.

48. Nardini L, Hunt RH, Dahan-Moss YL, Christie N, Christian RN, Coetzee M, Koekemoer LL, 2017. Malaria vectors in the Democratic Republic of the Congo: the mechanisms that confer insecticide resistance in Anopheles gambiae and Anopheles funestus. Malar J 16: 448.

49. Bayoh MN, Lindsay SW, 2003. Effect of temperature on the development of the aquatic stages of Anopheles gambiae sensu stricto (Diptera: Culicidae). Bull Entomol Res 93: 375-381.

50. Blanford JI, Blanford S, Crane RG, Mann ME, Paaijmans KP, Schreiber KV, Thomas MB, 2013. Implications of temperature variation for malaria parasite development across Africa. Sci Rep 3: 1300.

51. Carrion Martin Al, Bil K, Salumu P, Baabo D, Singh J, Kik C, Lenglet $A, 2014$. Mortality rates above emergency threshold in population affected by conflict in north Kivu, Democratic Republic of Congo, July 2012-April 2013. PLoS Negl Trop Dis 8: e3181.

52. Charchuk R, Paul MK, Claude KM, Houston S, Hawkes MT, 2016. Burden of malaria is higher among children in an internal displacement camp compared to a neighbouring village in the Democratic Republic of the Congo. Malar J 15: 431.
53. Melku M, Takele WW, Anlay DZ, Ekubagewargies DT, Getaneh Z, Abebe M, Abebe Z, 2018. Male and undernourished children were at high risk of anemia in Ethiopia: a systematic review and meta-analysis. Ital J Pediatr 44: 79.

54. Ballin A, Senecky Y, Rubinstein U, Schaefer E, Peri R, Amsel S, Vol $\mathrm{M}$, Amit $\mathrm{Y}$, Boaz M, 2012. Anemia associated with acute infection in children. Isr Med Assoc J 14: 484-487.

55. Ballin A, Lotan A, Serour F, Ovental A, Boaz M, Senecky Y, Rief S, 2009. Anemia of acute infection in hospitalized children-no evidence of hemolysis. J Pediatr Hematol Oncol 31: 750-752.

56. Abshire TC, Reeves JD, 1983. Anemia of acute inflammation in children J Pediatr 103: 868-871.

57. Teh RN, Sumbele IUN, Meduke DN, Ojong ST, Kimbi HK, 2018. Malaria parasitaemia, anaemia and malnutrition in children less than 15 years residing in different altitudes along the slope of Mount Cameroon: prevalence, intensity and risk factors. Malar J 17: 336.

58. Sifft KC et al., 2016. Asymptomatic only at first sight: malaria infection among schoolchildren in highland Rwanda. Malar $J$ 15: 553.

59. Okell LC, Bousema T, Griffin JT, Ouedraogo AL, Ghani AC, Drakeley CJ, 2012. Factors determining the occurrence of submicroscopic malaria infections and their relevance for control. Nat Commun 3: 1237.

60. Carneiro I, Roca-Feltrer A, Griffin JT, Smith L, Tanner M, Schellenberg JA, Greenwood B, Schellenberg D, 2010. Agepatterns of malaria vary with severity, transmission intensity and seasonality in sub-Saharan Africa: a systematic review and pooled analysis. PLoS One 5: e8988.

61. Shekalaghe SA, Bousema JT, Kunei KK, Lushino P, Masokoto A, Wolters LR, Mwakalinga S, Mosha FW, Sauerwein RW, Drakeley CJ, 2007. Submicroscopic Plasmodium falciparum gametocyte carriage is common in an area of low and seasonal transmission in Tanzania. Trop Med Int Health 12: 547-553.

62. Brazeau NF, Tabala M, Kiketa L, Kayembe D, Chalachala JL, Kawende B, Lapika B, Meshnick SR, Yotebieng M, 2016. Exclusive breastfeeding and clinical malaria risk in 6-month-old infants: a cross-sectional study from Kinshasa, Democratic Republic of the Congo. Am J Trop Med Hyg 95: 827-830.

63. Kalanda BF, Verhoeff FH, Brabin BJ, 2006. Breast and complementary feeding practices in relation to morbidity and growth in Malawian infants. Eur J Clin Nutr 60: 401-407.

64. Nankabirwa V, Tylleskar T, Nankunda J, Engebretsen IM, Sommerfelt H, Tumwine JK, PROMISE EBF Research Consortium, 2011. Malaria parasitaemia among infants and its association with breastfeeding peer counselling and vitamin $A$ supplementation: a secondary analysis of a cluster randomized trial. PLoS One 6: e21862.

65. World Health Organization, 2007. Evidence on the Long-Term Effects of Breastfeeding: Systematic Review and Meta-Analyses. Geneva, Switzerland: WHO. 